\title{
La notion de paysage-type
}

Lors d'un cours de perfectionnement pour enseignants sur les paysages-types de Suisse ${ }^{1}$, des notions centrales de la géographie ont été sommairement discutées: le terme de paysage, sa typologie, les types de paysages et les paysages-type. Il ne s'agit pas de chercher absolument des définitions valables, mais de faire quelques commentaires sur la problématique de ces notions. Ces considérations devraient aussi servir à discuter la manière de faire en recherchant des paysages-types pour l'enseignement et en préparant la documentation pour les élèves et les étudiants. Des dossiers de ce genre, résultats d'un cours antérieur, sont présentés par AERNI et STAUB (1982); d'autres ont été fournis par JEANNERET (1986 et 1989), BUMANN (1987) et ZURBRIGGEN (1988), d'autres sont en préparation.

\section{Paysage}

Le paysage en tant que terme géographique ne jouit pas partout de la même acceptation. Ainsi MOORE $\left(1968^{4}\right)$ renonce à un article intitulé «landscape» dans son "Dictionnary of Geography», 4e édition, tandis que MONKHOUSE (19702: 204), dans un même ouvrage, fait valoir une application artistique du terme «... used originally by artists to denote rural scenery (Dutch, landschap)».

En France, Pierre George (1974: 314), dans la 2e édition de son «Dictionnaire de la géographie», est encore très réservé: «Paysage», "employé par certaines écoles étrangères pour désigner le milieu naturel synthétique, objet d'une géographie physique globale». Dix ans plus tard, dans la 3e édition, la définition est déjà beaucoup plus étoffée, englobant les éléments physiques et humains (GEORGE 1984: 336): «Paysage». "...Il devient synonyme d'environnement dans le processus de perception de l'espace - se confond alors avec l'espace vécu.»

En produisant une «Géographie des paysages», Gabriel ROUGERIE (1977) admet la validité et l'importance du terme, en commençant son ouvrage par la

\footnotetext{
1 Cours no 8.08.11 "Typlandschaften der Schweiz" du Centre pour le perfectionnement des professeurs de l'enseignement secondaire et de l'Association Suisse des professeurs de géographie, tenu à Berne en janvier et mai 1988.
}

remarque: "Il est commode de définir la géographie comme l'étude des paysages.» (p. 3). De son côté, Olivier DOLLFUs relie le terme de paysage aussi à la notion de l'espace (1980: 6): «L'espace géographique est un espace changeant et différentié dont l'apparence visible est le paysage.» Enfin, WIEBER (1984) résume l'«Appréhension et compréhension des paysages» par la géographie française des vingt dernières années, tandis que BERTRAND (1984) analyse le rôle des paysages au cours de l'histoire de la géographie française récente et PINCHEMEL (et alii, 1987) propose un enseignement de la lecture du paysage. Pour la géographie d'expression allemande, le terme de «Landschaft» a toujours été appliqué et discuté, parfois même très contesté (voir PAFFEN 1973).

\section{Typologie des paysages}

Les géographes français n'ont pas été parmi les premiers à s'occuper d'une systématique de la surface terrestre et de ses phénomènes. CLOZIER, dans son «Histoire de la Géographie» (1972), ne fait pas état d'une telle préoccupation dans le passé, et pour CLAVAL (1982), il n'en est non plus question dans le cadre d'une «Nouvelle Géographie». «La géographie des paysages» de ROUGERIE (1977) constitue par contre une typologie globale, même si le terme luimême n'est pas appliqué. Dollfus (1980) utilise non seulement le terme de "type de paysage», mais aussi «la famille des régions», qui "sont des espaces rendus homogènes, à un titre ou un autre». Ses «types d'organisation de l'espace géographique» rejoignent l'idée de la géographie volontaire.

Il semble que c'est surtout depuis Alexandre de HUMBOLDT que la géographie générale a tenté de devenir une science systématique et explicative, selon l'affirmation de LAUTENSACH 1953 (dans STORKEBAUM 1967: 549): "Vor allem seit A. vom HUMBOLDT hat sich die Allgemeine Geographie im Sinn einer Typen bildenden und damit systematische Elemente

François Jeanneret, lecteur/chargé de cours, Les Vernes 4 2534 Orvin 
enthaltenden Wissenschaft entwickelt." Bien entendu, c'est la systématique de la biologie qui a servi de modèle. Mais nombreux sont les critiques mettant en question l'analogie entre les deux branches. Citons LAUTENSACH 1953 (dans STORKEBAUM 1967: 559): "Dass eine klassifikatorische Systematisierung von geographischen Räumen unmöglich oder zumindest unzweckmässig ist.» Puis il précise que le terme d'espèce ne s'applique guère à un paysage: «Im übrigen stehen Species und Subspecies von Natur getrennt nebeneinander ganz im Gegensatz zur Geographie, deren Gegenstände, die irdischen Räume, ein Kontinuum bildet.» L'article de LAUTENSACH est d'ailleurs intitulé «Typus und Individuum». GEORGE (1978) est du même avis: "La géographie est l'étude d'un espace continu.» Voici l'obstacle majeur à toute tentative de typologie. SCHMITTHENNER (1951: danS STORKEBAUM 1967: 212), en discutant la classification proposée par OBST (1950 dans STORKEBAUM 1967: 159-187), ne peut pas accepter de types de paysages autrement qu'en tant que définitions pragmatiques: «Die Typen der Landschaft sind als klassifizierendes System keine Realität, sondern pragmatische Definitionen.» L'Américain HARTSHORNE (1933 dans WINKLER 1975: 205) est par contre catégorique: "Areas cannot be classified logically according to their total character.» Si nous suivons les considérations de KIRSCH et alii. (1986: 214/215, 143), l'établissement d'une typologie des paysages est une démarche essentiellement géomorphologique, ce qui est en contradiction avec DOLlFus (1980), en particulier ses «types d'organisation de l'espace géographique».

Le fondateur de la géographie des paysages («Landschaftskunde») du début du siècle, Siegfried PAsSARGE (théorie résumée en 1933), a aussitôt introduit l'élément de la comparaison («géographie comparée des paysages» = «Vergleichende Landschaftskunde»). Par contre, LAUTENSACH propose dès 1953, en se basant sur le caractère continu du paysage, le concept des «variations géographiques des paysages» («geographischer Formenwandel») qualifié par LESER (1976: 191) de contribution la plus remarquable dans la discussion des systèmes naturels.

Quelques tentatives de quantifier les caractéristiques de paysages ont été faites. Citons l'exemple de la nordicité ou degré de nordicité exprimé en valeur polaire VAPO développé par HAMELIN (1968) pour l'Arctique et adapté par CAILleuX (1972) à l'Antarctique. La valeur polaire est la somme de dix indices partiels physiques (latitude, climat, végétation) et humains (accessibilité, population, activité économique). Une telle démarche serait valable pour toute typologie basée sur le principe de facteurs dominants.

Parmi les exemples les plus récents, citons BEAULIEU-GARNIER (1971) qui propose, après une discussion de l'homogénéité et de la régionalisation, une typologie de la division de l'espace. GROSJEAN (1973) fournit un relevé et une systématique des physiotopes du canton de Berne. Les écoles de géographie s'orientant vers la géo-écologie ne renoncent pas à des typologies. DEMANGEOT (1985) parle de milieux «naturels» et LESER (1976) de types d'espace («Raumtypen»). Les approches sont différentes et variées, mais tous ces auteurs évitent le terme de paysage!

\section{Types de paysages et paysages-types}

Toute démarche de typologie va aboutir, d'une manière ou d'une autre, à la définition de types de paysages, donc en répondant à certains critères. Chaque paysage individuel sera analysé par rapport aux critères choisis, son appartenance à un type va être déterminée ainsi.

Dans leur enseignement, certains géographes se limitent à des exemples de paysages choisis pour étudier les types. Il y aurait donc lieu de définir le paysage-type - terme faisant défaut dans les dictionnaires et lexiques de la géographie - comme représentant ou exemple d'un type de paysage (= Die Typlandschaft ist ein Vertreter oder ein Beispiel eines Landschaftstypes). Le paysage-type est une nécessité didactique et méthodologique pour remplacer ou compléter la géographie régionale. Il sera un exemple offrant un certain nombre voire un maximum de propriétés caractéristiques du type de paysage concerné. Mais il est aussi pensable de traiter un cas de transition, puisque la majorité des types de paysage ne peuvent pas être délimités avec précision.

Parmi les rares auteurs à utiliser le terme de paysage-type, citons BRUNEAU et COURADE (1984: 309) qui, dans le cadre de la discussion de la tropicalité, reprennent des considérations de Pierre GOuRou: "Ces paysages-structures ne sont pas les paysages réels observés au sol d'un point précis, ou délimités sur une vue verticale aérienne ou de satellites par un photo-interprète, ce sont des paysages-types, abstraits, reconstruits par le géographe et valables pour une aire régionale donnée... Ces paysages-structures ou paysagesmodèles à l'échelle locale ou régionale permettent le mieux d'étudier les rapports hommes-milieux naturels et de se référer au concept de civilisation dont les techniques sont le principe explicatif des paysages." LEBEAU (1979), par contre, dans son ouvragé sur les types de structures agraires, illustre ses théories de manière générale par les exemples qu'il appelle des paysages-types. Toutefois, ceux-ci ne sont cependant pas présentés de manière globale, dans le sens d'une typologie des paysages.

Souvent, dans la recherche, l'étude de cas est au début de vastes programmes. Les régions analysées par EWALD (1978) par rapport à l'évolution des paysages constituent un exemple. L'auteur utilise le terme de paysage-type sans le définir ou le discuter (p. 213). La délimitation de ces espaces est d'ail- 
leurs parfaitement arbitraire puisqu'il s'agit chaque fois d'une feuille de la Carte nationale de la Suisse (au 25000 ). Un autre exemple sont les aires-test du programme MAB («Man and Biosphere») de l'UNESCO (décrites par exemple par PORTMANN 1983). En effet, de petites régions représentent de vastes espaces offrant un certain nombre de similitudes.

Des paysages-types sont le plus souvent présentés dans un but didactique. En Suisse, une des premières publications a été la collection de feuilles extraites de l'Atlas topographique (carte SIEGFRIED), sélectionnée et commentée par voSSELER (1928). Les tableaux scolaires suisses (séries de géographie) et la «Suisse vue d'avion» (BINGGELI 1989, sous-titré: «Paysages types sur transparents quadricolores») présentent un choix de paysage-types, comme certains manuels et ouvrages illustrés. Voici quelques exemples: les «Panoramas aériens» édités par KUHN et alii (1973), les «Géographies de la Suisse» de BAER (19885: p. 79-154 «Choix de paysages») et de WIESLI (1986: p. 191-278 «Einzellandschaften»).

Si le terme de paysage-type reflète un concept et une réalité tant pour l'enseignement que pour la recherche géographique, ne faudrait-il pas l'appliquer d'une manière systématique dans le cadre des préoccupations des géographes?

\section{Zusammenfassung: Der Begriff der Typenlandschaft}

Typenlandschaften werden in der Geographie - im Unterricht wie in der Forschung - immer wieder herangezogen, obwohl der Begriff noch kaum diskutiert worden ist. Schon der Begriff der Landschaft ist umstritten, gelegentlich wird er einfach vermieden. Der vorliegende Artikel ist ein Beitrag zur Diskussion im Rahmen der französisch-, deutschund englischsprachigen Literatur. Dabei ist das Problem der Landschafttypologie unvermeidbar, denn schließlich kann eine Typlandschaft nur ein Vertreter oder ein Beispiel eines Landschaftstypes sein.

\section{Summary: The term of type landscape}

Type landscapes are often used in Geography - in teaching as well as in research -, although the term has not yet been generally discussed. The term of landscape itself is rather controversial, its use is sometimes carefully avoided. This paper is a contribution to the discussion dealing with a choice of aspects covered by the litterature in French, in German and in English. However, the problem of the typology of landscapes cannot be left out, as a type landscape can only set an example or represent a landscape type.

\section{Literatur}

AERNI, K./STAUB, B. (1982): Landschaftsökologie im Geographieunterricht. Heft 1 und 2 (Vier geographische Praktikumsaufgaben für Mittelschulen). Geographica Bernensia S8 + S9, Bern: $62+119 p$.

BAER, O. (1988, 5): Géographie de la Suisse. Delta \& Spes Denges: $246 \mathrm{p}$.

BERTRAND, G. (1984): Les géographes français et leurs paysages. Ann. de géographie, Paris, 515: 218-229.

BINGGELI, V. (1980): La Suisse vue d'avion. Paysages types sur transparents quadricolores. Ingold Herzogenbuchsee: $74 \mathrm{p}$.

BINGGELI, V./FEIGENWINTER, M. (1980): Die Schweiz vom Flugzeug aus. Typlandschaften auf farbigen Transparenten. Ingold Herzogenbuchsee: $181 \mathrm{p}$.

BRUNEAU, M./COURADE, G. (1984): Existe-t-il une géographie humaine tropicale? A la recherche d'un paradigme de Pierre Gourou. I'Espace Géographique 4 (1984) Paris: 306-316.

BUMAN, P. (1987): Leuk und das Rhonetal. Fiche de géographie 2. Office fédéral de topographie. Association suisse des professeurs de géographie, Geographica Bernensia Berne: $4 p$.

CAILLEUX, A. (1972): Indices polaires en Antarctique et Subantarctique. Annales de Géographie 81(445), Paris: p. 257-277.

CHAPMAN, G. P. (1977): Human and Environmental Systems. A. Geographer's Appraisal. Academic Press, London: $421 \mathrm{p}$.

CLAVAL, P. (1982, 2): La nouvelle géographie. Que sais-je? No 1693, Presses universitaires de France, Paris: 128 p.

CLOZIER, R. (1972, 5): Histoire de la géographie. Que saisje? No 65. Presses universitaires de France, Paris: $128 \mathrm{p}$.

DEMANGEOT, J. (1985, 2): Les milieux «naturels» du globe. Collection Géographie, Masson, Paris: 250 p.

DOLLFUS, O. (1980, 3): L'espace géographique. Que saisje? No 1390, Presses universitaires de France, Paris: $128 \mathrm{p}$.

EWALD, K. C. (1978): Der Landschaftswandel. Zur Veränderung schweizerischer Kulturlandschaften im 20. Jahrhundert. Eidg. Anstalt für das forstliche Versuchswesen Birmensdorf. Bericht 191: Tätigkeitsbericht der Naturforschenden Gesellschaft Baselland, Band 30, Liestal: p. 55-308 + 14 cartes.

GEORGE, P. (1974, 2/1984, 3): Dictionnaire de la géographie. Presses universitaires de France, Paris: 485 p. (1984).

GEORGE, P. (1978, 2): Les méthodes de la géographie. Que sais-je? No 1398, Presses universitaires de France, Paris: $128 \mathrm{p}$.

GROSJEAN, G. et al. (1973): Historische Planungsgrundlagen - Bases historiques de l'aménagement. Planungsatlas - Atlas de l'aménagement t. 3. Berne: 328 p. +5 cartes.

GUERMOND, Y. (1977): Analyse de Systèmes en Géographie. Presses universitaires de Lyon: $324 \mathrm{p}$.

HAMELIN, L.-E. (1968): Un indice circumpolaire. Ann. de Géographie 422, Paris: p. 413-430.

HARTSHORNE, R. (1939): The Nature of Geography. Annals of the Association of American Geographers: p. 173-658.

HAGGETT, P. (1983, 3): Geography - A Modern Synthesis. Harper \& Row, New York: $643 \mathrm{p}$. 
JEANNERET, F. (1980): Grosses Moos. Kommentar zum Schweizerischen Schulwandbildwerk. Bild 188. Ingold Herzogenbuchsee: $30 \mathrm{p}$.

JEANNERET, F. (1986): Goldau. Fiche de géographie 1. Office fédéral de topographie. Association suisse des professeurs de géographie, Geographica Bernensia Berne: 4 p.

JEANNERET, F. (1989): Val-de-Travers. Fiche de géographie 3. Office fédéral de topographie. Association suisse des professeurs de géographie, Geographica Bernensia Berne: 4 p.

KIRSCH, H./MAURER, R./SCHMIDT-KOEHL, W./SCHULZ, K.NOELZING, O. (1981): Geographie. Fachbegriffe der Geographie. Studienbücher Diesterweg + Sauerländer, Frankfurt a. M. + Aarau $188+232$ p.

KUHN, W. et al. (1973): Schweiz - Suisse - Switzerland. Flugpanorama - Panorama aérien - Aerial Panorama. Lang, Berne: $112 \mathrm{p}$.

LEBEAU, R. (1979): Les grands types de structures agraires dans le monde. Initiation aux études de géographie. Masson. Paris: $162 \mathrm{p}$.

LESER, H. (1976): Landschaftsökologie. UTB 521. Ulmer Stuttgart: $432 \mathrm{p}$.

MONKHOUSE, T. J. $(1970,2)$ : A Dictionnary of Geography Arnold, London: $378 \mathrm{p}$.

MOORE, W. G. (1968, 4): A Dictionnary of Geography. Penguin Reference Books Harmondsworth: $234 \mathrm{p}$.

PAFFEN, K. (1973): Das Wesen der Landschaft. Wege der Forschung XXXIX, Wissenschaftliche Buchgesellschaft Darmstadt: $514 \mathrm{p}$.

PASSARGE, Siegfried (1933): Einführung in die Landschaftskunde. Teubner, Leipzig: $99 \mathrm{p}$.
PINCHEMEL, Ph., et alii (1987): Lire les paysages. Documentation photographique no 6088 (Avril 1987). La Documentation Française, Paris: $42 \mathrm{p}$.

PORTMANN, J.-P. (1984): L'homme et son environnement: le programme MAB. Bull. de la Soc. Neuchâteloise de Géographie 28, Neuchâtel: 147-176.

ROUGERIE, G. (1977, 2): La nouvelle géographie. Que saisje? No 1693. Presses universitaires de France, Paris: $128 \mathrm{p}$.

STORKEBAUM, W. (1987): Zum Gegenstand und zur Methode der Geographie. Wege der Forschung LVIII, Wissenschaftliche Buchgesellschaft Darmstadt: $632 \mathrm{p}$.

VOSSELER, P. (1928): Die Landschaften der Schweiz. 20 Blätter aus dem topographischen Atlas der Schweiz. Eidg. Landestopographie Bern: 20 cartes +2 feuilles $+64 p$

WENDT, B. (1978): Geographie - Gegenstand und Methode. Eine wissenschaftlich-propädeutische Einführung. Ploetz Didaktik 1, Ploetz Freiburg + Würzburg: 109 p.

WIEBER, J.-C. (1984): Appréhension et compréhension des paysages. La recherche géographique française. Comité national français de géographie, XXVe congrès international de géographie Paris-Alpes 1984: p. 91-97.

WIESLI, U. (1986): Die Schweiz. Wissenschaftliche Länderkunde Bd. 26. Wissenschaftliche Buchgesellschaft Darmstadt: 354 p. +3 cartes +27 photos.

WINKLER, E. (1976): Probleme der Allgemeinen Geographie. Wege der Forschung CCIC. Wissenschaftliche Buchgesellschaft Darmstadt: $429 \mathrm{p}$.

ZURBRIGGEN, B. (1988): Fribourg. Fiche de géographie 4. Office fédéral de topographie. Association suisse des professeurs de géographie, Geographica Bernensia Berne: 4 p. 